\title{
Envelhecimento populacional e gastos com internação do SUS: uma análise realizada para o Brasil entre 2000 e 2010
}

\author{
Cristiano Sathler dos Reis* \\ Kenya Noronha* \\ Simone Wajnman ${ }^{\star \star \star}$
}

\begin{abstract}
A população mundial vem experimentando um processo gradativo de envelhecimento de sua estrutura etária, em função da queda acentuada da fecundidade e da mortalidade nas últimas décadas. Esse processo, que está em curso em praticamente todos os países do mundo, traz à tona a preocupação com o crescimento das despesas em saúde. 0 objetivo do presente artigo é avaliar o efeito das mudanças da estrutura etária nos gastos com internação do Sistema Único de Saúde do Brasil. Para tanto, foi realizada uma decomposição dos gastos em saúde, utilizando o método Tchoe e Nam, adaptado para a realidade do caso brasileiro. A decomposição também é feita considerando três grupos de doenças: infecciosas e parasitárias, neoplasias e circulatórias. Os dados são provenientes do Sistema de Informações Hospitalares (SIH-SUS). De forma geral, os resultados encontrados mostram a importância da variação na estrutura etária (envelhecimento populacional) nos gastos das internações hospitalares do SUS entre 2000 e 2010, principalmente para os grupos de doenças associados a uma população mais envelhecida, doenças circulatórias e neoplasia.
\end{abstract}

Palavras-chave: Envelhecimento populacional. Gastos com internação. Decomposição.

\footnotetext{
* Centro de Desenvolvimento e Planejamento Regional (Cedeplar), Universidade Federal de Minas Gerais (UFMG), Belo Horizonte-MG, Brasil (cristiano.sathler@gmail.com).

** Centro de Desenvolvimento e Planejamento Regional (Cedeplar), Universidade Federal de Minas Gerais (UFMG), Belo Horizonte-MG, Brasil (knoronha@cedeplar.ufmg.br).

${ }^{\star \star \star}$ Centro de Desenvolvimento e Planejamento Regional (Cedeplar), Universidade Federal de Minas Gerais (UFMG), Belo Horizonte-MG, Brasil (simonewajnman@gmail.com).
} 


\section{Introdução}

A população mundial vem experimentando um processo gradativo de envelhecimento de sua estrutura etária, em função da queda da fecundidade e da mortalidade. 0 segmento de idosos (60 anos ou mais) no mundo alcançou 770 milhões de pessoas em 2010, cerca de $11 \%$ da população. Esse contingente deve chegar a 1 bilhão em 2020 (aproximadamente $13 \%$ da população mundial), dos quais $20 \%$ residirão em países em desenvolvimento (U.S. CENSUS BUREAU, 2010).

No Brasil, a partir de 1940, houve uma queda significativa das taxas de mortalidade, enquanto a fecundidade se manteve inalterada, resultando em uma população jovem e com altas taxas de crescimento (CHAIMOWICZ, 1997). Desde o final da década de 1960, a fecundidade passou a cair vertiginosamente, sobretudo nos grupos populacionais com maior renda e nas áreas urbanas das regiões mais desenvolvidas do país (CARVALHO; WONG, 2008). Os resultados do Censo Demográfico de 2010 indicam que a taxa de fecundidade total (TFT), que representa o número médio de filhos que uma mulher teria ao final do período reprodutivo, era de aproximadamente 1,9 filho por mulher no país, contra 2,9 em 1991 e 6,2 filhos em 1940 (IBGE, 2001, 2011). Essa diminuição da taxa de fecundidade determinou um rápido processo de envelhecimento da população brasileira.

0 processo de transição demográfica é caracterizado por uma redução da população jovem e aumento daquela pertencente aos grupos etários mais avançados. No Brasil, a participação de crianças de 0 a 14 anos na população total diminuiu entre 2000 e 2010 (de $29,6 \%$ para $24,1 \%$ ), enquanto a dos grupos mais velhos aumentou. A população de 65 anos ou mais passou de 3,1\%, em 1970, para 7,4\%, em 2010, ou seja, quase dobrou (IBGE, 2011), e estima-se que esse percentual alcançará 24\% em 2050 (IBGE, 2013). Esse processo vem sendo observado em decorrência, principalmente, de quedas acentuadas das taxas de fecundidade observadas nas últimas décadas no país.

De acordo com o IBGE (2011), o índice de envelhecimento da população, conforme demonstram as projeções, deve continuar sofrendo transformações nas próximas décadas. Em 1980, para cada grupo de 100 crianças de 0 a 14 anos, havia apenas 10,5 idosos de 65 anos ou mais de idade no país. Ao longo das décadas, este índice sofreu variações positivas, passando de 13,9, em 1991, para 19,8, em 2000, e para 30,7 idosos em 2010. As projeções sugerem ganhos ainda mais consideráveis no índice de envelhecimento brasileiro após a virada do milênio, devendo alcançar um patamar de 47,7 em 2020, 76,4 em 2030, 113,2 em 2040 e 160,90 em 2050 (IBGE, 2013).

Simultaneamente a essas mudanças, observa-se no país um processo de transição epidemiológica que, de acordo com Omran (1971), engloba três mudanças básicas: substituição das doenças transmissíveis por doenças não transmissíveis e causas externas; deslocamento da carga de morbi-mortalidade dos grupos mais jovens para os grupos mais idosos; e transformação de uma situação em que predomina a mortalidade para outra na qual a morbidade é dominante (OMRAN, 1971). 
Tais alterações modificam significativamente o perfil de saúde da população, com impactos importantes sobre os gastos com saúde. Ao contrário de processos agudos que se resolvem rapidamente por meio da cura ou do óbito, tornam-se predominantes as doenças crônicas em decorrência do envelhecimento populacional, que muitas vezes significam vários anos de utilização do serviço de saúde, como medicamentos, consultas médicas, internações de longa duração, resultando em maiores gastos (WONG; CARVALHO, 2006). Além disso, o tratamento dessas doenças exige a utilização de serviços de maior complexidade, com alto nível tecnológico (BILGEL; TRAN, 2013).

0 aumento dos gastos em saúde, observado tanto em países desenvolvidos como naqueles em desenvolvimento, tem atraído considerável atenção dos gestores de políticas públicas e de pesquisadores da área de saúde. Os fatores determinantes desse crescimento têm sido amplamente explorados na literatura internacional (HITIRIS; POSNETT, 1992; SMITH; HEFFLER; FREELAND, 2000; CUTLER; MEARA, 2001; XUKE; SAKSENAE HOLLY, 2011) e nacional (SILVESTRE, 2001; KILSZTAJN et al., 2002; NUNES, 2003; BERENSTEIN; WAJNMAN, 2008). De maneira geral, o envelhecimento populacional vem se destacando entre as variáveis que explicam o aumento destes gastos.

O consumo de cuidados com a saúde não é uniformemente distribuído ao longo do ciclo de vida dos indivíduos. A curva de custo médio em função da idade apresenta um formato de "U", sendo mais alto entre as crianças e idosos e menor para a população jovem. Normalmente, a taxa de utilização dos serviços de saúde é mais elevada para os indivíduos com idade superior a 65 anos (RICHARDSON; ROBERTSON, 1999; MILLER, 2001; ARISTE; CAR, 2002; IOM, 2008).

0 maior gasto com serviços de saúde entre os idosos está associado ao perfil de morbidade deste grupo populacional, caracterizado pela maior prevalência de doenças crônico-degenerativas. Além disso, as taxas de internação para grupos idosos são mais elevadas. Esse grupo populacional tem maior probabilidade de ser internado, consumindo mais serviços de saúde (GLENNERSTER; MATSAGANIS, 1994; RECHEL et al., 2009), e apresenta custos médios de internações mais altos quando comparado com os grupos mais jovens (RUBIO, 1990). Assim, como estas doenças estão diretamente relacionadas com a idade e o estilo de vida, a composição demográfica de uma população torna-se um aspecto central para a análise dos gastos com saúde de uma sociedade (POL; THOMAS, 2000).

Além do envelhecimento, outros fatores relacionados a esse processo também contribuem para aumentar os gastos com saúde, tais como mudanças tecnológicas (FUCHS, 1998), nível de renda (NEWHOUSE, 1977) e proximidade à morte (LUBITZ; PRIHODA, 1984). Muito embora o objetivo desse artigo seja apenas avaliar em que medida mudanças na estrutura etária afetam os gastos com saúde, é importante salientar que parte desse efeito pode estar associada à interação desses fatores com o envelhecimento e gastos com saúde (HITIRIS; POSNETT, 1992; DI MATTEO, 2005). Ignorar essa relação e a contribuição desses fatores para explicar as mudanças observadas nos gastos pode sobre-estimar o efeito do processo de envelhecimento populacional. Sociedades mais 
envelhecidas, em geral, apresentam nível de renda e de desenvolvimento econômico mais elevado e, consequentemente, maior propensão para incorporação de novas tecnologias no setor de saúde (WIENER; TILLY, 2002; DAVIS et al., 2007). Ademais, evidências empíricas têm salientado a importância da proximidade à morte na determinação dos gastos com saúde (LUBITZ; RILEY, 1993; HIMSWORTH; GOLDACRE, 1999). Quanto mais próximo à morte, maiores são os gastos com saúde. Como a taxa de mortalidade é mais elevada entre os idosos, o maior nível de gastos neste segmento populacional pode estar mais associado à proximidade à morte do que à idade propriamente dita (BREYER; FELDER, 2006; BERENSTEIN, 2009).

0 presente estudo pretende investigar o efeito das mudanças demográficas sobre os gastos de internação do Sistema Único de Saúde do Brasil, por meio do método de decomposição desenvolvido por Tchoe e Nam (2010), o qual permite decompor a diferença dos gastos totais de internação entre dois anos em quatro efeitos: preço (medido pela variação dos gastos médios por idade); taxa (calculada pela variação das taxas de internação por idade); composição etária (definida pela alteração da estrutura etária); e mudança do tamanho populacional.

A análise de decomposição é realizada para o Brasil entre 2000 e 2010. A decomposição é conduzida para o total de internações e para três grupos distintos de doenças: neoplasias, doenças do aparelho circulatório e doenças infecciosas e parasitárias. As duas primeiras estão associadas a populações mais envelhecidas enquanto as doenças infecciosas e parasitárias relacionam-se a grupos mais jovens (LIMA-COSTA et al., 2000; LUNA, 2002; OMRAN, 2005; LIMA-COSTA; MATOS, 2008).

\section{Metodologia}

\section{Base de dados}

A principal fonte de informações utilizada neste estudo é o Sistema de Informações Hospitalares (SIH), que contém registros administrativos da Autorização de Internação Hospitalar (AIH) do Sistema Único de Saúde (SUS). Os registros são disponibilizados pelo Ministério da Saúde, sendo processados e consolidados pelo departamento de informática do SUS (Datasus). O SIH fornece informações sobre o número e o valor total das internações, que estão disponibilizadas segundo diferentes atributos, tais como ano, município de residência e de realização da internação, tipo de procedimento realizado, grupos de doença da CID, sexo e idade. Além do SIH, são também utilizados dados do Censo Demográfico do Instituto Brasileiro de Geografia e Estatística (IBGE), que fornece o total da população por sexo e grupo etário em cada ano analisado.

A análise compreende o período de 2000 a 2010. As informações das AlH estão disponíveis a partir de 1995 e somente em 1998 os procedimentos passaram a ser codificados segundo a 10 10 revisão da CID. Com isso, ao restringir a análise a partir de 2000, evitamos 
fazer a compatibilização entre a 9a e a $10^{\underline{a}}$ classificação de doenças, o que poderia comprometer a análise histórica. Além disso, as informações sobre a população estudada estão disponíveis para 2000 e 2010, anos da realização do Censo Demográfico no país, o que torna possível a estimação mais acurada das taxas de internação, dispensando o uso de estimativas populacionais.

Para a realização da decomposição entre 2000 e 2010, os valores dos gastos com internação para 2010 são deflacionados com base no Índice Nacional de Preço ao Consumidor Amplo (IPCA), tendo como base o ano de 2000. Os resultados são estimados, primeiro, considerando homens e mulheres conjuntamente e, depois, desagregando por sexo. As internações por parto e gravidez são excluídas da análise devido à grande concentração desse tipo de internação entre mulheres na faixa etária de 15 a 49 anos, podendo sobre-estimar o efeito da estrutura etária (PINHEIRO et al., 2002; NUNES, 2002; KILSZTAJN et al., 2002; BERENSTEIN; WAJNMAN, 2008).

A cada internação realizada no âmbito do SUS é preenchida uma AIH, que é composta por diversas informações referentes às internações, como identificação e qualificação do paciente, procedimentos, exames e atos médicos realizados, diagnóstico, valores incorridos, entre outros. As unidades hospitalares do SUS enviam aos gestores municipais, por meio da $\mathrm{AlH}$, as informações das internações efetuadas. Estes dados são processadas no Datasus, gerando os créditos referentes aos serviços prestados. O SIH é considerado uma relevante fonte de informações, com registros sistemáticos mensais desagregados por indivíduos e hospitais, além de fornecer subsídios tanto para o planejamento e gestão de pesquisas epidemiológicas (estudos de mortalidade/morbidade, prevenção e promoção à saúde, avaliação da qualidade dos serviços), quanto para fiscalização e auditoria do Ministério da Saúde (MARINHO; MORENE; CAVALINI, 2001; SÁ, 2006; BERENSTEIN; WAJNMAN, 2008).

Por considerar apenas internações hospitalares, a análise realizada nesse estudo se restringe aos serviços de maior complexidade, que em geral estão associados a pacientes cujo estado de saúde é mais grave (BERENSTEIN; WAJNMAN, 2008). Ademais, a fonte de dados utilizada compreende informações sobre as internações hospitalares financiadas pelo SUS, excluindo as que são financiadas pelo setor privado. No entanto, essa restrição não compromete a amplitude e a relevância das conclusões obtidas por esse trabalho, uma vez que as internações do SUS possuem grande representatividade no número de internações do país e consomem mais da metade do montante de recursos destinados à saúde (BOGUE; HALL; LA FORGIA, 2007; RODRIGUES, 2010; PAIM et al., 2011; RODRIGUES; AFONSO, 2012). Segundo dados da Pesquisa Nacional de Saúde de 2013, as internações do SUS representam cerca de $66 \%$ de todas as internações do país (IBGE, 2013).

Uma dificuldade das informações provenientes do SIH-SUS decorre da possibilidade de o preenchimento das AlH ser feito de forma incorreta ou incompleta, seja por falta de treinamento do profissional que o realiza, seja pela existência de tentativas de fraude ao SUS (dupla cobrança de procedimentos, alteração de dados sobre o paciente internado, entre outros). Dessa forma, a qualidade dos registros pode estar comprometida. No 
entanto, diversos estudos realizados em diferentes localidades no país têm mostrado que as informações, especialmente aquelas relacionadas ao diagnóstico principal, apresentam grau elevado de confiabilidade. Veras e Martins (1994) verificaram para o Rio de Janeiro, em 1986, que os dados relacionados ao diagnóstico principal podem ser utilizados com uma confiabilidade de mais de $80 \%$. Estudo semelhante de Mathias e Soboll (1998), para o Município de Maringá (PR) em 1992, mostra que a utilização do banco de dados da AlH possui uma confiabilidade satisfatória para diagnósticos mais frequentes na população. Escosteguy et al. (2002), analisand o como diagnóstico principal o infarto agudo do miocárdio no Município do Rio de Janeiro em 1997, revelam que a maior limitação foi o elevado sub-registro do diagnóstico secundário da AlH.

A qualidade do SIH vem se aperfeiçoando a cada ano, com melhorias técnicas que identificam e corrigem tais distorções. Assim, estudos realizados com essa fonte de dados possuem grande poder explicativo sobre as condições relacionadas às internações hospitalares realizadas pelo SUS (MARINHO; MORENE; CAVALINI, 2001; RODRIGUES, 2010).

\section{Definição das variáveis}

As variáveis utilizadas para a realização da análise de decomposição são o gasto médio de internação, taxa de internação e população total. As equações (1) e (2) definem algebricamente cada um desses indicadores. Tais medidas são calculadas por grupos etários quinquenais, sexo, ano e pelos três grupos de doenças.

As internações são classificadas por grupos etários quinquenais, com o primeiro sendo definido apenas pelos indivíduos menores de um ano e o último por aqueles com 80 anos e mais. Para os grupos de doença, as internações foram classificadas de acordo com a metodologia proposta pela CID-10 tal como disponibilizado pelo Datasus.

As informações sobre gasto total e número de internações fornecidas pelo SIH-SUS podem ser obtidas por município de residência ou de ocorrência da internação. Dado que é utilizada como referência a população residente, são consideradas as internações por município de residência.

A taxa de internação é definida pela razão entre o número de internações hospitalares e o total da população para cada grupo etário quinquenal $i$ :

$$
T I_{i}=\frac{N_{i}}{P_{i}}
$$

Onde:

$T I_{i}=$ taxa internação hospitalar para o grupo etário quinquenal $i$;

$N_{i}=$ número de internações para o grupo etário quinquenal $i$;

$P_{i}=$ população total em cada grupo etário quinquenal $i$.

Ressalta-se que, como os gastos analisados referem-se às internações ocorridas no setor público cujo acesso é universal, optou-se por utilizar a população total como proxy da população potencialmente coberta pelo SUS. 
A equação (2) define o gasto médio de internação $(G M)$ em um dado grupo etário quinquenal $i$. De acordo com essa equação, o GM é igual ao gasto total da internação sobre o número total de internações:

$G M_{i}=\frac{G T_{i}}{N_{i}}$

Onde:

$G M_{i}=$ gasto médio de internação para o grupo etário quinquenal $i$;

$G T_{i}=$ gasto total das internações para o grupo etário quinquenal $i$;

$N_{i}=$ número de internações para o grupo etário quinquenal $i$;

O gasto total das internações (GT) é constituído pela soma do valor total das AlH e compreende os gastos incorridos com todos os serviços relativos a cada internação hospitalar, tais como serviços profissionais, serviços auxiliares de diagnose e terapia, gastos com recém-nascidos, órtese e prótese, tomografias e ressonância magnética, transfusão de sangue, transplantes, analgesia obstétrica e de pediatria.

\section{Método}

A metodologia de análise baseia-se no método proposto por Tchoe e Nam (2010), adaptado para a realidade do caso brasileiro. 0 método consiste na decomposição da diferença dos gastos hospitalares entre dois pontos no tempo. Neste artigo, foram considerados os anos de 2000 e 2010. Esse método permite a identificação de quatro componentes que explicam a trajetória dos gastos com internações no período de análise: variação de preço obtido a partir da variação dos gastos médios (efeito-preço); variação na taxa de internação (efeito-taxa); mudança na estrutura etária (efeito composição etária ou efeito envelhecimento); e crescimento populacional.

Com essa análise, é possível verificar o quanto do crescimento dos gastos hospitalares entre 2000 e 2010 é explicado por cada um desses componentes. 0 exercício é realizado para a população total e por sexo, considerando o total das internações e desagregando por grupos de doenças. Para facilitar o entendimento e a descrição da análise de decomposição, a exposição dos procedimentos metodológicos é feita para o total das internações e sem distinguir por sexo. A aplicação da análise por grupos de doenças e por sexo é direta.

0 ponto de partida da análise de decomposição é definir o gasto total com internação da seguinte forma:

$\mathrm{GT}=\sum_{i=0}^{80+} P_{i} \times T I_{i} \times G M_{i}$

Onde:

GT = gasto total com internação;

$P_{i}=$ população total em cada grupo etário quinquenal $i$;

$T I_{i}=$ taxa de internação para o grupo etário quinquenal $i$ conforme definido pela equação (1); $G M_{i}=$ gasto médio com internação para o grupo etário quinquenal $i$ conforme definido pela equação (2). 
Dessa maneira, a variação dos gastos com internação entre 2000 e 2010 pode ser definida da seguinte forma:

$$
\Delta G T=\sum_{i=0}^{80+} \Delta P_{i} \times \Delta T I_{i} \times \Delta G M_{i}
$$

Onde:

$\Delta G T=G T^{10}-G T^{00}$ representa a variação do gasto total entre 2010 (10) e 2000 (00);

$\Delta G M_{i}=G M_{i}^{10}-G M_{i}^{00}$ representa a variação do gasto médio no grupo etário $i$ entre 2010 (10) e $2000(00)$.

$\Delta T I_{i}=T I_{i}^{10}-T I_{i}^{00}$ representa a variação da taxa de internação no grupo etário $i$ entre 2010 (10) e $2000(00)$.

$\Delta P_{i}=P_{i}^{10}-P_{i}^{00}$ representa a variação do tamanho populacional no grupo etário $i$ entre 2010 (10) e 2000 (00).

A variação do gasto médio com internação $(\Delta G M)$ e da taxa de internação $(\Delta T I)$ é determinada tanto pelo envelhecimento populacional - que eleva o peso relativo dos grupos etários de maior gasto médio - como também por outros fatores - que alteram os gastos médios em cada grupo etário, tais como mudanças tecnológicas e crescimento econômico (TCHOE; NAM, 2010). Neste artigo, o objetivo é entender em que medida o envelhecimento da estrutura etária explica a variação dos gastos totais $(G T)$ com internação. Para a realização dessa análise, é necessário, portanto, isolar o efeito da mudança na estrutura etária presente nos dois componentes da variação do gasto total ( $\triangle G M$ e $\Delta T I$ ) observada entre 2000 e 2010 . As equações (5) e (6) decompõem a variação na taxa de internação $(\Delta T I)$ e no gasto médio $(\triangle G M)$ em dois componentes, de forma a isolar esse efeito:

$\Delta T I=\Delta E E^{T I} \times \Delta T I^{a}$

$\Delta G M=\Delta E E^{G M} \times \Delta G M^{a}$

Onde:

$\triangle E E^{T I}=$ mudança na estrutura etária incidindo sobre as taxas de internação por grupos de idade entre 2000 e 2010;

$\Delta T I^{a}=$ variação da taxa de internação ajustada entre 2000 e 2010;

$\Delta E E^{G M}=$ mudança na estrutura etária incidindo sobre os gastos médios com internação por grupos de idade entre 2000 e 2010;

$\Delta G M^{a}=$ variação do gasto médio com internação ajustado entre 2000 e 2010.

As mudanças no gasto médio ajustado $\left(\Delta G M^{a}\right)$ e na taxa de internação ajustada $\left(\Delta T I^{a}\right)$ entre os dois anos analisados referem-se à variação das taxas e gastos médios dos quais se expurgam o efeito de composição da estrutura etária. Esses dois componentes correspondem ao efeito preço e efeito taxa, respectivamente.

As variáveis $\triangle E E^{T I}$ e $\triangle E E^{G M}$ representam o efeito da alteração na estrutura etária (envelhecimento populacional) entre dois pontos no tempo (2000 e 2010) sobre as taxas de internação e sobre os gastos médios. Substituindo as equações (5) e (6) em (4), temos: $\Delta G T=\sum_{i=0}^{80+} \Delta P_{i} \times\left(\Delta E E_{i}^{T I} \times \Delta T I_{i}^{a}\right) \times\left(\Delta E E_{i}^{G M} \times \Delta G M_{i}^{a}\right)$ 
A taxa de variação do gasto total é obtida utilizando-se o logaritmo natural de cada componente da equação (7), que permite identificar os quatro componentes da análise de decomposição proposta. Os termos $\Delta G M^{a}$ (efeito preço) e $\Delta T I^{a}$ (efeito taxa) correspondem à parcela da variação total dos gastos entre 2000 e 2010 que se deve à mudança no gasto médio e na taxa de internação, respectivamente, expurgados os efeitos da mudança na composição etária. 0 termo $\Delta P_{i}$ refere-se à parcela explicada pelo crescimento populacional. Finalmente, o termo $\triangle E E$ representa a parcela da variação no gasto total explicada pelo efeito de mudanças da composição etária (envelhecimento populacional). Este componente $\triangle E E$ corresponde à soma do efeito do envelhecimento populacional sobre a taxa de internação $\triangle E E^{T I}$ e sobre o gasto médio $\triangle E E^{G M}$. A distinção do efeito da estrutura etária sobre dois componentes (taxa de internação e gasto médio) é importante, pois os perfis etários da taxa de internação e do gasto médio por idade são diferenciados e, portanto, a mudança da estrutura etária afeta cada um desses componentes de forma distinta. ${ }^{1}$

0 efeito do envelhecimento populacional na taxa de internação $\left(\Delta E E^{T I}\right)$ é estimado conforme definido pela equação (8):

$\Delta E E^{T I}=\frac{\sum_{i=0}^{80+} P_{i}^{10} \times \frac{P^{00}}{P^{10}} \times T I_{i}^{00}}{\sum_{i=0}^{80+} P_{i}^{00} \times T I_{i}^{00}}$

Essa equação mostra em quanto a taxa de internação se alteraria devido apenas a mudanças na estrutura etária da população (envelhecimento populacional). Como pode ser observado, esse efeito controla pelo crescimento populacional $\left(\frac{p^{00}}{p^{10}}\right)$ e mantém constante a taxa de internação no nível observado em 2000. De forma análoga, o efeito de mudança da estrutura etária nos gastos médios é estimado da seguinte maneira:

$\Delta E E^{G M}=\frac{\sum_{i=0}^{80+} T I_{i}^{10} \times \frac{T I^{00}}{T I^{10}} \times G M_{i}^{00}}{\sum_{i=0}^{80+} T I_{i}^{00} \times G M_{i}^{00}}$

Nesse caso, mantém-se constante o gasto médio no nível observado em 2000 e mensura-se a variação desses gastos decorrente apenas de mudanças na estrutura etária da taxa de internação.

0 efeito taxa $\left(\Delta T I^{a}\right)$ e o efeito preço $\left(\Delta G M^{a}\right)$ são obtidos com base nas informações das variações na taxa de internação $(\Delta T I)$ e no gasto médio $(\Delta G M)$, e do efeito da mudança na estrutura etária ( $\triangle E D^{T I}$ e $\left.\triangle E D^{G M}\right)$, como definido pelas equações (5) e (6), respectivamente.

\footnotetext{
${ }^{1}$ A probabilidade de os indivíduos se internarem depende de sua condição de saúde e da oferta de serviços para seu tratamento. Os gastos médios com saúde, por sua vez, são afetados tanto por esses fatores como também pelo conteúdo tecnológico do cuidado provido e a forma como os serviços são precificados no mercado.
} 


\section{Resultados}

\section{Análise descritiva}

Essa seção descreve o comportamento dos três componentes da decomposição dos gastos de internação hospitalar (população, taxa de internação e gasto médio de internação) para 2000 e 2010. A análise é realizada para o total de internações.

O Gráfico 1 mostra a distribuição da população brasileira por grupos quinquenais de idade para 2000 e 2010. Ao longo dos dez anos, houve mudança na forma das curvas que representam a estrutura etária da população. Em ambos os sexos, a proporção de jovens se reduziu com o concomitante aumento da participação de idosos.

\section{GRÁFICO 1}

Pirâmide etária, por sexo

Brasil - 2000-2010

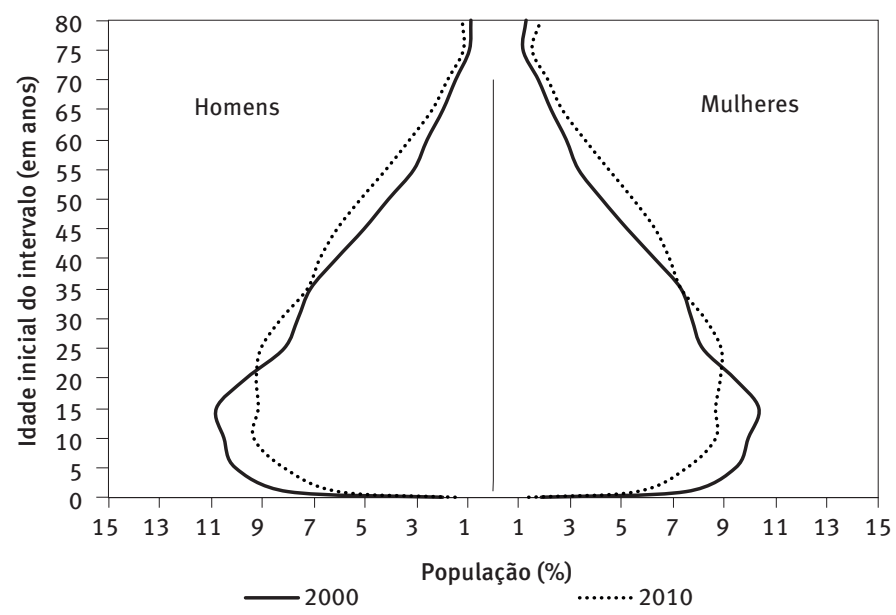

Fonte: IBGE. Censos Demográficos 2000 e 2010.

O Gráfico 2 apresenta informações sobre o gasto médio por internação em 2000 e 2010, para homens, mulheres e total, segundo grupos de idades. Observa-se que o comportamento do gasto médio por internação é similar para as três desagregações. Os gastos médios foram altos para o grupo com menos de um ano de idade, reduziram-se para as faixas etárias subsequentes ( 1 a 4 até 10 a 14 anos) e aumentaram a partir dos 15 anos, passando a função de gasto a apresentar um formato de "U" invertido e crescendo até alcançar 69 anos. Entre os indivíduos acima de 70 anos, os gastos passam a apresentar trajetória descendente.

Esse comportamento do gasto médio é observado em outros estudos (RUBIO, 1990; LIMA-COSTA; BARRETO; GIATTI, 2003; LOYOLA-FILHO et al., 2004). Uma provável explicação para o decréscimo dos gastos médios é a redução da eficácia de determinados procedimentos mais onerosos para as pessoas pertencentes aos grupos etários mais velhos, que, teoricamente, responderiam menos ao tratamento, já que possuem condição de saúde mais frágil (NUNES, 2004). Entre os dois anos de análise, foi observada uma distribuição do gasto médio total por 
internação muito similar. No entanto, para todos os grupos etários, o gasto médio por internação em 2010 foi maior em relação a 2000, principalmente para as faixas de idade extremas.

\section{GRÁFICO 2}

Gasto médio total por dia de internação para homens, mulheres e total da população, segundo grupos etários

Brasil - 2000-2010
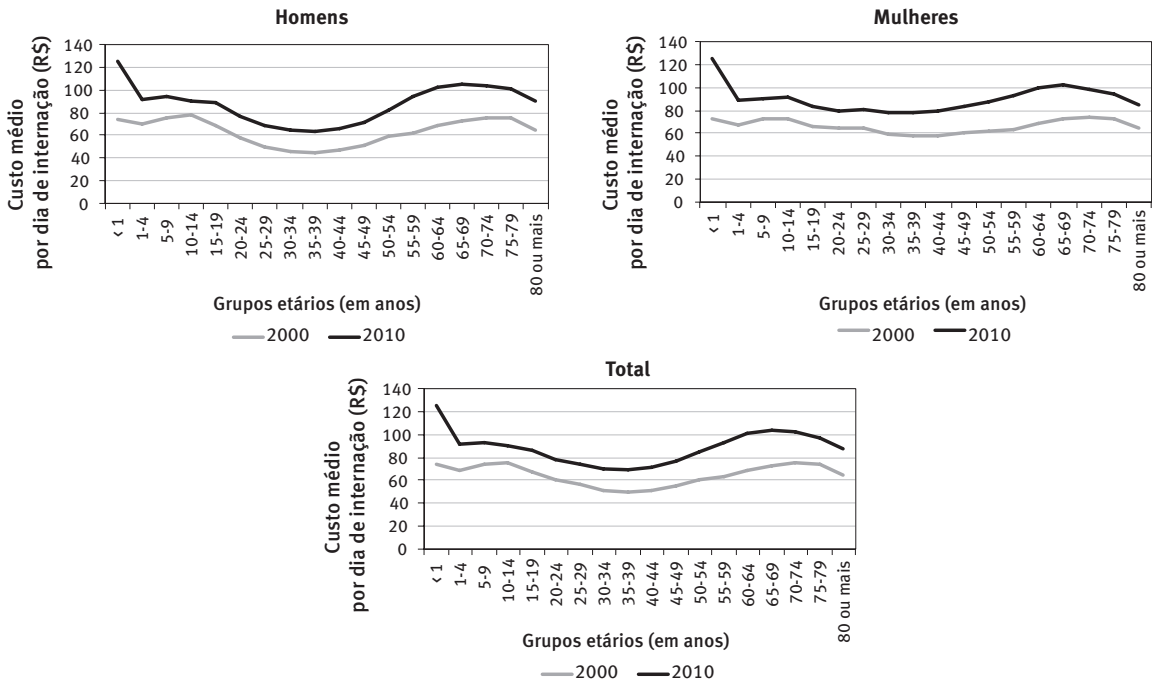

Fonte: Ministério da Saúde. Sistema de Informações Hospitalares - SIH (Datasus); IBGE. Censos Demográficos 2000 e 2010.

GRÁFICO 3

Taxas de internação para homens, mulheres e total da população, segundo grupos etários Brasil - 2000-2010
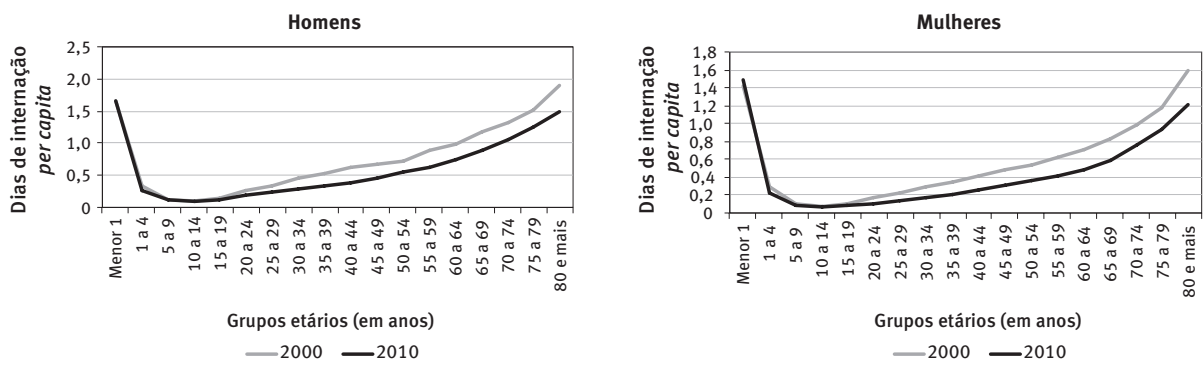

$-2000-2010$

Grupos etários (em anos)

Total $-2000-2010$

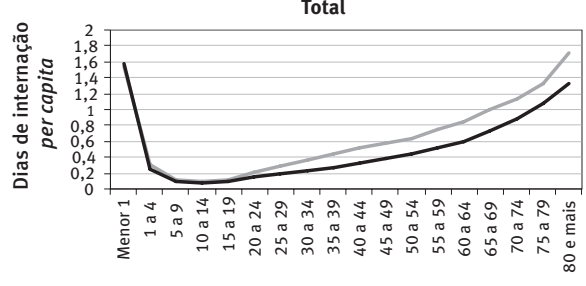

Grupos etários (em anos)

$-2000-2010$

Fonte: Ministério da Saúde. Sistema de Informações Hospitalares - SIH (Datasus); IBGE. Censos Demográficos 2000 e 2010. 
O Gráfico 3 apresenta informações sobre as taxas de internações. Independentemente do sexo analisado, as taxas são nitidamente mais elevadas para os grupos etários extremos, assumindo um formato de "U", em conformidade com o observado na literatura existente (KILSZTAJN et al., 2002; NUNES, 2004; RIBEIRO, 2005). Para ambos os sexos, verifica-se que as taxas de internações para 2010 são menores em todos os grupos etários em relação a 2000.

\section{Análise de decomposição}

A Tabela 1 traz a decomposição do gasto de internação hospitalar entre 2000 e 2010 para ambos os sexos e total de internação, segundo três grupos de doenças: infecciosas e parasitárias, neoplasias e doenças do aparelho circulatório. De forma geral, todas as decomposições realizadas tiveram um crescimento dos gastos com internação no período 2000-2010.

A decomposição dos gastos com internação para o total das internações demonstra uma participação significativa do efeito preço e da composição etária. De acordo com a Tabela 1, o efeito preço respondeu por $63,4 \%$ para o sexo masculino, $63,6 \%$ para o feminino e $64,7 \%$ para o total da população. Já o efeito composição etária contribuiu para o crescimento dos gastos hospitalares principalmente para mulheres (77\%), seguidas pelo total da população $(61,2 \%)$ e homens $(50,7 \%)$. Por outro lado, a análise para o total das internações evidencia uma contribuição negativa do efeito taxa para explicar as diferenças nos gastos de internação hospitalar entre os dois anos. Esse comportamento é resultado da queda das taxas de internação entre 2000 e 2010, conforme mostra o Gráfico 3.

A contribuição de cada componente para explicar as diferenças no gasto com internação hospitalar depende do grupo de doença analisado. No caso das neoplasias, o maior responsável para explicar a diferença nos gastos totais entre os dois anos foi o efeito composição etária (32,1\%), seguido pelo efeito taxa (31,5\%). Para os homens, a principal proporção da diferença atribuída foi o efeito taxa (42,9\%), enquanto para as mulheres foi o efeito composição etária (36,3\%).

Para as doenças do aparelho circulatório, o efeito composição etária respondeu pela maior parte do crescimento dos gastos com internação, contribuindo com $80,9 \%$ para o sexo masculino, 122,9\% para o feminino e $97,3 \%$ para o total. Assim como na decomposição para o total de internações, o efeito taxa foi negativo para todas as decomposições das doenças do aparelho circulatório. Observa-se que esse efeito taxa negativo ocorre apenas para esse grupo de doenças, afetando o efeito taxa total. A queda das taxas de internação total pode ser explicada, em grande parte, pela redução das internações por doenças do aparelho circulatório entre 2000 e 2010.

A análise de decomposição para as doenças infecciosas e parasitárias demonstra a importância do efeito preço, resultado da variação do gasto médio entre 2000 e 2010, contribuindo, em grande parte, para o crescimento dos gastos em saúde em todas as 
decomposições. Por outro lado, o efeito composição etária foi responsável por um aumento insignificante desse crescimento, sendo de apenas $1 \%$ para o sexo masculino, $3,4 \%$ para o feminino e 2,1\% para o total da população. Esse resultado sugere neutralidade do envelhecimento populacional com relação às internações por essa causa de doença. Isso ocorre porque, para esse grupo de morbidade, a taxa de internação e os gastos médios são, em geral, mais elevados nos grupos de idade extremos (crianças e idosos). Dessa forma, o envelhecimento populacional, ao reduzir a proporção de crianças e elevar a de idosos, produz efeitos que tendem a se compensar.

TABELA 1

Decomposição dos gastos de internação, por grupos de doenças, segundo sexo e componentes Brasil - 2000-2010

\begin{tabular}{|c|c|c|c|c|}
\hline Sexo e componentes & Total & Neoplasias & $\begin{array}{c}\text { Doenças do } \\
\text { aparelho } \\
\text { circulatório }\end{array}$ & $\begin{array}{c}\text { Infecciosas } \\
\text { e } \\
\text { parasitárias }\end{array}$ \\
\hline \multicolumn{5}{|l|}{ Homens } \\
\hline Crescimento populacional & 45,3 & 15,8 & 30,3 & 26,1 \\
\hline Composição etária total & 61,2 & 28,5 & 86,4 & 9,5 \\
\hline Composição etária - efeito taxa & 34,5 & 21,5 & 55,8 & $-0,02$ \\
\hline Composição etária - efeito preço & 26,7 & 7,01 & 30,6 & 9,5 \\
\hline Dias de internação per capita ajustado (efeito taxa) & $-126,2$ & 22,1 & $-62,1$ & $-25,0$ \\
\hline Custo por dia de internação ajustado (efeito preço) & 119,6 & 33,5 & 45,4 & 89,5 \\
\hline Total (\%) & 100,0 & 100,0 & 100,0 & 100,0 \\
\hline \multicolumn{5}{|l|}{ Mulheres } \\
\hline Crescimento populacional & 59,6 & 24,4 & 47,5 & 24,4 \\
\hline Composição etária total & 91,7 & 34,9 & 148,8 & 10,7 \\
\hline Composição etária - efeito taxa & 45,5 & 28,9 & 83,3 & $-3,7$ \\
\hline Composição etária - efeito preço & 46,2 & 6,0 & 65,5 & 14,3 \\
\hline Dias de internação per capita ajustado (efeito taxa) & $-168,3$ & $-1,9$ & $-138,2$ & 0,9 \\
\hline Custo por dia de internação ajustado (efeito preço) & 117,0 & 42,5 & 41,9 & 64,1 \\
\hline Total (\%) & 100,0 & 100,0 & 100,0 & 100,0 \\
\hline \multicolumn{5}{|l|}{ Total } \\
\hline Crescimento populacional & 51,4 & 19,8 & 36,8 & 25,4 \\
\hline Composição etária total & 73,3 & 31,4 & 110,0 & 10,0 \\
\hline Composição etária - efeito taxa & 38,8 & 25,1 & 66,2 & $-1,8$ \\
\hline Composição etária - efeito preço & 34,5 & 6,2 & 43,7 & 11,8 \\
\hline Dias de internação per capita ajustado (efeito taxa) & $-143,5$ & 11,3 & $-91,6$ & $-12,9$ \\
\hline Custo por dia de internação ajustado (efeito preço) & 118,7 & 37,6 & 44,8 & 77,6 \\
\hline Total (\%) & 100,0 & 100,0 & 100,0 & 100,0 \\
\hline
\end{tabular}

Fonte: Ministério da Saúde. Sistema de Informações Hospitalares - SIH (Datasus); IBGE. Censos Demográficos 2000 e 2010.

\section{Discussão}

Os resultados de decomposição encontrados no presente estudo mostram a importância da variação na estrutura etária nos gastos das internações hospitalares do SUS, entre 2000 e 2010, exceto para o grupo de doenças infecciosas e parasitárias. Para o total de 
internações, o efeito composição etária foi responsável por 61,2\% dos aumentos dos gastos com internações. Ao analisar a decomposição total por sexo, o efeito da composição etária contribuiu com $50,7 \%$ e $77 \%$ dos gastos com internações realizadas entre os homens e mulheres, respectivamente.

Esses resultados diferem daqueles encontrados por Berenstein e Wajnman (2008), Tchoe e Nam (2010) e Dunn, Liebman e Shapiro (2012). Berenstein e Wajnman (2008) realizaram a decomposição considerando as diferenças nos gastos com internações em Curitiba e Belém para pacientes do sexo masculino em 2004. 0 efeito composição etária (dado pela variação da estrutura etária) foi responsável por apenas $12,7 \%$ da diferença dos gastos com internação, uma vez que as estruturas etárias são bem semelhantes entre as capitais. Tchoe e Nam (2010), em estudo realizado para a Coreia do Sul entre 1991 e 2003, observaram que o efeito composição etária contribui com menos de $10 \%$. Dunn, Liebman e Shapiro (2012), em estudo realizado para os EUA, também encontraram uma pequena contribuição do efeito composição etária para explicar as diferenças nos gastos hospitalares entre 2003 e 2007. A variação do efeito composição etária entre os três estudos pode ser resultado de variações de métodos aplicados, contextos, períodos analisados e regiões distintas, composição etária e padrões epidemiológicos diferentes para cada análise de decomposição.

A análise das taxas de internação no Brasil mostra uma queda acentuada entre $2000 \mathrm{e}$ 2010, evidenciada na análise de decomposição dos gastos para o total de internações, já que o efeito taxa foi negativo para o total da população e para ambos os sexos. A redução das taxas de internação pode ser justificada pela diminuição do número de leitos observada nos últimos anos, pelo estabelecimento de cotas mensais de internação e pelas políticas de saúde adotadas recentemente no Brasil focalizando a atenção primária (RODRIGUES, 2010; CARNEIRO et al., 2013; BERENSTEIN, 2005; BERENSTEIN; WAJNMAN, 2008). Segundo dados do Cadastro Nacional de Estabelecimentos de Saúde (CNES), o Brasil contava, em 2005, com um total de 375.600 leitos hospitalares, passando para 327.600 leitos em 2012 , uma redução de $12,8 \%$. Além disso, como forma de conter o crescimento nos custos de serviços hospitalares, foram adotadas cotas de internação no país. A partir de 1994 , a quantidade de AlH autorizadas foi limitada em $10 \%$ da população de cada localidade, ocorrendo decréscimos ao longo dos anos: em 1995, o limite máximo de AlH passou para $9 \%$ e, em 2002, foi fixado em 8,7\% (RODRIGUES, 2010). Segundo Neto et al. (2008), as restrições orçamentárias explicam boa parte da retração das taxas de internações, dadas as mudanças na legislação que definiram novos parâmetros de distribuição das AlH.

Com relação às mudanças nas políticas de saúde, observa-se que o Ministério da Saúde tem focado seus esforços na ampliação do cuidado primário. Tais esforços têm sido notados, principalmente, desde a implementação em 1994 da Estratégia de Saúde da Família (ESF), que tem como objetivo melhorar o acesso da população ao cuidado da atenção primária, com ênfase no cuidado preventivo, em detrimento da lógica hospitalocêntrica até então experimentada no país (CASTRO; TRAVASSOS; CARVALHO, 2005; PAIM, 2008; MENDES, 
2009; CONASS, 2011; ANDRADE et al., 2013). 0 foco no cuidado preventivo tende a reduzir complicações e agudizações relacionadas a doenças crônicas, tais como hipertensão arterial e diabetes, contribuindo para diminuir a demanda por internações hospitalares e serviços de maior complexidade (MENDES, 2009). Segundo dados do Ministério da Saúde, a cobertura populacional da Equipe de Saúde da Família aumentou de 6,6\%, em 1998, para 52,2\%, em 2010. O número de equipes da Saúde da Família era de 0,3 por mil habitantes em 1994 e chegou a 31,6 por mil em 2010. Essa forte expansão apresenta impacto significativo no acesso e uso da atenção primária em saúde, com potencial de reduzir as internações por condições sensíveis à atenção primária (ICSAP) (MACINKO et al., 2011; BOING et al., 2012).

Existem evidências empíricas mostrando a importância da cobertura da ESF para a redução das ICSAP no Brasil (MACINKO; GUANAIS; SOUZA, 2003; CAMPOS; THEME-FILHA, 2012; RASELLA et al., 2014). Ceccon, Meneghel e Viecili (2014) avaliam as ICSAP e a cobertura populacional da ESF no Brasil entre 1998 e 2006. Segundo os autores, ficam evidentes os decréscimos das ICSAP no Brasil, corroborando com pesquisas realizadas em outros países, em que foi apontada relação entre a redução das ICSAP e a ampliação da atenção primária (CAMINAL et al., 2004; SANCHEZ et al., 2008). Em outro estudo regional, Rodrigues-Bastos et al. (2014) observam uma queda significativa dessa taxa de internações em Minas Gerais, passando de 20,75 para 14,92 internações por mil habitantes, entre 2000 e 2010. Segundo os autores, as taxas de internação por hipertensão arterial, asmas e diabetes sofreram a maior retração no período estudado.

A análise do gasto médio para o total de internações revela crescimento entre $2000 \mathrm{e}$ 2010 para todos os grupos etários, principalmente entre os jovens e idosos. Esse resultado vai ao encontro da análise de decomposição, já que o efeito preço teve importante contribuição, respondendo por $63,4 \%$ para o sexo masculino, $63,6 \%$ para o feminino e $64,7 \%$ para o total da população. 0 crescimento dos gastos médios hospitalares, entre 2000 e 2010 , pode ser em decorrência da incorporação de novas tecnologias em produtos, procedimento e práticas clínicas (CUTLER; MCCLELLAN, 2001; CHERNEW; NEWHOUSE, 2011). A incorporação de novas tecnologias, em geral, substitui tratamentos padrões por outros mais complexos e permite oferecer tratamento a pacientes que antes não poderiam ser tratados de maneira segura (DAVIS et al., 2007). Além disso, há um aumento na demanda de pacientes e provedores por essa inovação e, consequentemente, um maior gasto em saúde (WEISBROD, 1991).

A análise por grupos de doenças revela resultados importantes. Para os dois grupos de doenças associados a uma população mais envelhecida - neoplasias e doenças do aparelho circulatório -, o efeito composição etária foi o principal responsável pelo crescimento nos gastos hospitalares do SUS. Por outro lado, causas de morbi-mortalidade por doenças infecciosas e parasitárias, que possuem maior prevalência em locais no início da transição epidemiológica, apresentam pequeno efeito da estrutura etária, respondendo por apenas $1 \%$ do aumento dos gastos hospitalares no período de 2000 a 2010 para o sexo masculino, $3,4 \%$ para o feminino e $2,1 \%$ para o total da população. De forma geral, o efeito preço (dado 
pela variação do gasto médio entre 2000 e 2010) é maior para as doenças infecciosas em relação às neoplasia e doenças do aparelho circulatório. A principal razão por essa diferença entre os grupos de doenças analisados, quando se observa o total da população, é que o crescimento dos gastos médios entre 2000 e 2010 foi maior para as doenças infecciosas e parasitárias ( $42 \%$, passando de $R \$ 284,1$ para $R \$ 405,5)$ em comparação às doenças do aparelho circulatório (39\%, de $R \$ 724,2$ para $R \$ 1.013,4)$ e neoplasias $(19 \%$, de $R \$ 718,5$ para $\mathrm{R} \$ 854,8)$. Além disso, como dito anteriormente, o efeito composição etária possui uma grande participação para as decomposições das doenças do aparelho circulatório e neoplasias, o que, de certa forma, minimiza o peso do efeito preço.

É importante destacar a proporção negativa da diferença atribuída ao efeito taxa na decomposição dos gastos hospitalares por doenças do aparelho circulatório. Esse efeito taxa negativo é resultado da queda das taxas de internações desse grupo de doenças no período analisado. Em estudo realizado no Paraná entre 2000 e 2011, os resultados sugerem que o aumento da cobertura da Estratégia Saúde da Família foi fator relevante para a diminuição das internações por condições cardiovasculares (LENTSCK; MATHIAS, 2015).

Quando se observa a decomposição do gasto total entre homens e mulheres, notam-se alguns diferenciais importantes. Para a decomposição das doenças do aparelho circulatório e as infecciosas e parasitárias, o efeito taxa é muito menor para mulheres $(-123,7)$ do que para homens $(-60,5)$. A principal razão dessa diferença é a queda mais acentuada da taxa de internação feminina em relação à masculina. Já para as neoplasias, o maior efeito taxa pode ser observado para os homens, resultado da maior queda da taxa de internação masculina entre 2000 e 2010 em relação à feminina. Por fim, as decomposições para as doenças infecciosas e parasitárias possuem um padrão semelhante entre os sexos.

\section{Considerações finais}

O objetivo desse trabalho foi analisar a importância do envelhecimento da população para explicar as variações nos gastos de internação pública para o Brasil. Foram realizadas decomposições para avaliar a diferença nos gastos de internação entre 2000 e 2010, para ambos os sexos e para o total da população. A decomposição foi feita considerando o total de internações e desagregando para três grupos distintos de doenças: neoplasias, doenças do aparelho circulatório e doenças infecciosas e parasitárias. Ao decompor o crescimento dos gastos hospitalares em efeito preço, efeito taxa, efeito composição etária e crescimento populacional, verificou-se que o efeito composição etária (envelhecimento populacional) exerce papel determinante nas diferenças de gasto total entre 2000 e 2010 , com exceção do grupo de doenças associado a uma população mais jovem - doenças infecciosas e parasitárias. Ainda, o efeito preço também explica de maneira significativa o crescimento dos gastos hospitalares para o total da população e para os grupos de doenças do aparelho circulatório e infecciosas e parasitárias. Dessa forma, o aumento da proporção de idosos poderia gerar um crescimento expressivo nos custos, especialmente para grupos 
de doenças que são mais prevalentes nessa faixa de idade, tais como as do aparelho circulatório e neoplasias. Políticas de saúde que foquem em cuidados preventivos e na adoção de tecnologias e procedimentos que reduzem os custos dos tratamentos podem contribuir para conter essa ampliação dos gastos com internação. Além disso, é importante repensar as formas de cuidados para as doenças crônicas, reduzindo o foco nas internações como principal alternativa de tratamento para as pessoas idosas.

\section{Referências}

ANDRADE, M. V. et al. Desigualdade socioeconômica no acesso aos serviços de saúde no Brasil: um estudo comparativo entre as regiões brasileiras em 1998 e 2008. Economia Aplicada, v. 17, n. 4, p. 623-645, 2013

ARISTE, R.; CARR, J. New considerations on empirical analysis of the determinants of Canadian provincial government health expenditures, 1966-1998. Canada: Health Demand and Supply Analysis Division, August 2, 2002 (Working paper, n. 02-06).

BERENSTEIN, C. K. O perfil etário dos custos de internação na saúde pública no Brasil: uma análise para as capitais das regiões metropolitanas do Brasil em 2000. 2005. 91 f. Dissertação (Mestrado em Demografia) - Centro de Desenvolvimento e Planejamento Regional, Universidade Federal de Minas Gerais (Cedeplar/UFMG), Belo Horizonte, 2005.

Os efeitos de idade e proximidade à morte sobre os gastos com internações no SUS: evidências com base no caso de Minas Gerais, 2004/2005. 2009. 95 f. Tese (Doutorado em Demografia) - Centro de Desenvolvimento e Planejamento Regional, Universidade Federal de Minas Gerais (Cedeplar/UFMG), Belo Horizonte, 2009.

BERENSTEIN, C. K.; WAJNMAN, S. Efeitos da estrutura etária nos gastos com internação na saúde pública: uma análise de decomposição para duas áreas metropolitanas brasileiras. Cadernos de Saúde Pública, Rio de Janeiro, v. 24, n. 10, p. 2301-2313, outubro 2008.

BILGEL, F.; TRAN, K. C. The determinants of Canadian provincial health expenditures: evidence from dynamic panel. Applied Economics, v. 45, n. 2, p. 201-212, 2005.

BOGUE, R. J.; HALL, C. H.; LA FORGIA, G. M. Hospital governance in Latin America - Results from a four nation survey. Washington, DC: World Bank, April 2007 (Health, Nutrition and Population Discussion Paper).

BOING, A. F.; VICENZI, R. F.; MAGAJEWSKI, F.; BOING, A. C.; MORETTI-PIRES, R. O.; PERES, K. G.; LINDNER, S. R.; PERES, M. R. Redução das internações por condições sensíveis à atenção primária no Brasil entre 1998-2009. Revista de Saúde Pública, v. 46, n. 2, p. 359-366, 2012.

BREYER, F.; FELDER, S. Life expectancy and health care expenditures: a new calculation for Germany using the costs of dying. Health Policy, v. 75, n. 2, p.178-186, 2006.

CAMPOS, A. Z.; THEME-FILHA, M. M. Internações por condições sensíveis à atenção primária em Campo Grande, Mato Grosso do Sul, Brasil, 2000 a 2009. Cadernos de Saúde Pública, v. 28, n. 5, p. 845-55, 2012.

CAMINAL, J.; STARFIELD, B.; SANCHEZ. E.; CASANOVA, C.; MORALES, M. The role of primary care in preventing ambulatory care sensitive conditions. European Journal of Public Health, v. 14, n. 3, p. 246-51, 2004.

CARNEIRO et al. Envelhecimento populacional e os desafios para o Sistema de Saúde brasileiro. São Paulo: Instituto de Estudos de Saúde Suplementar - IESS, 2013. 
CARVALHO, J. A. M.; WONG, L. L. R. A transição da estrutura etária da população brasileira na primeira metade do século XXI. Cadernos de Saúde Pública, v. 24, n. 3, p. 597-605, mar. 2008.

CASTRO, M. S. M.; TRAVASSOS, C.; CARVALHO, M. S. Efeito da oferta de serviços de saúde no uso de internações hospitalares no Brasil. Revista de Saúde Pública, v. 39, n. 2, p. 277-84, 2005.

CHAIMOWICZ, F. A saúde dos idosos brasileiros às vésperas do século XXI: problemas, projeções e alternativas. Revista de Saúde Pública, v. 31, n. 2, p. 184-200, abr. 1997.

CONSELHO NACIONAL DE SECRETÁRIOS DE SAÚDE. Atenção primária e promoção da saúde. 1. ed. Brasília: Conass, 2011 (Coleção Para Entender a Gestão do SUS).

CUTLER, D. M.; MEARA, E. The concentration of medical spending: an update. Themes in the economics of aging. University of Chicago Press, 2001. p. 217-240.

DATASUS - Departamento de Informática do SUS. Acesso em: 20 abr. 2013.

DAVIS, K. et al. Slowing the growth of U.S. health care expenditures: what are the options? [S.I.]: The Common Wealth Fund, 2007.

DI MATTEO, L. The macro determinants of health expenditure in the United States and Canada: assessing the impact of income, age distribution and time. Health Policy, v. 71, p. 23-42, 2005.

DUNN, A.; LIEBMAN, E.; SHAPIRO, A. H. Decomposing medical-care expenditure growth. Federal Reserve Bank of San Francisco, November 2012 (Working Paper Series).

ECOSTEGUY, C. C. et al. O Sistema de Informações Hospitalares e a assistência ao infarto agudo do miocárdio. Revista de Saúde Pública, v. 36, n. 4, p. 491-9, 2002.

FERnANdeS, A. A. Envelhecimento e saúde. Revista Portuguesa de Saúde Pública, v. 23, n. 2, 2005.

FUCHS, V. R. Health care for the elderly: how much? Who will pay for it? Cambridge, Mass.: National Bureau of Economic Research, 1998 (Working paper, n. 6755).

GLENNERSTER, H.; MATSAGANIS, M. The English and Swedish care reforms. International Journal of Health Services, v. 24, n. 2, p. 232-251, 1994.

HALL, B. H.; KHAN, B. Adoption of new technology. In: JONES, D. New economy handbook. New York: Academic Press, 2002.

HIMSWORTH, R. L.; GOLDACRE, M. J. Does time spent in hospital in the final 15 years of life increase with age at death? A population based study. British Medical Journal, n. 319, p. 1338-1339, Nov. 1999. Disponível em: 〈http://www.bmj.com/cgi/reprint/319/7221/1338〉. Acesso em: 23 maio 2013.

HITIRIS, T.; POSNETT, J. The determinants and effects of health expenditure in developed countries. Journal of Health Economics, n. 11, p. 173-181, 1992.

IBGE - Instituto Brasileiro de Geografia e Estatística. Censo Demográfico 2000. Rio de Janeiro: IBGE, 2001.

Projeção da população do Brasil por sexo e idade para o período 1980 a 2050 Revisão 2008. Rio de Janeiro: IBGE, 2008.

Projeção da população do Brasil por sexo e idade para o período 2000 a 2060 Revisão 2013. Rio de Janeiro: IBGE, 2013.

Censo Demográfico 2010. Rio de Janeiro: IBGE, 2011.

Pesquisa Nacional de Saúde 2013: percepção do estado de saúde, estilos de vida e doenças crônicas. Rio de Janeiro: IBGE, 2014. 
IOM - Institute of Medicine. Retooling for an aging America: building the health care workforce. Washington, DC: The National Academies Press, 2008.

KILSZTAJN, S.; ROSSBACH, A.; CAMARA, M. B.; CARMO, M. S. N. Serviços de saúde, gastos e envelhecimento da população brasileira. In: ENCONTRO NACIONAL DE ESTUDOS POPULACIONAIS, 13, Ouro Preto, 2002. Anais... Belo Horizonte: Abep, 2002 (CD-ROM).

LIMA-COSTA, M. F. et al. Diagnóstico da situação de saúde da população idosa brasileira: um estudo da mortalidade e das internações hospitalares públicas. Informe Epidemiológico do SUS, v. 9, n. 1, p. 23-41, 2000.

LIMA-COSTA, M. F.; MATOS, D. L. Tendências das condições de saúde e uso de serviços de saúde da população idosa brasileira: 20 anos de Sistema Único de Saúde. Saúde Brasil 2008: 20 anos de Sistema Único de Saúde (SUS) no Brasil. Brasília, DF: Ministério da Saúde, 2009.

LIMA-COSTA, M. F.; BARRETO, S. M.; GIATTI, L. Condições de saúde, capacidade funcional, uso de serviços de saúde e gastos com medicamentos da população idosa brasileira: um estudo descritivo baseado na Pesquisa Nacional por Amostra de Domicílios. Cadernos Saúde Pública, v. 19, n.3, p. 735-743, jun. 2003.

LENTSCK, M. H.; MATHIAS, T. A. F. Internações por doenças cardiovasculares e a cobertura da estratégia saúde da família. Rev. Latino-Am. Enfermagem, v. 23, n. 4, p. 611-9, jul./ago. 2015.

LOYOLA FILHO, A. I. et al. Causas de internações hospitalares entre idosos brasileiros no âmbito do Sistema Único de Saúde. Epidemiologia e Serviços de Saúde, v. 13, n. 4, p. 229-238, dez. 2004.

LUBITZ, J.; PRIHODA, R. The use and costs of medicare services in the last 2 years of life. Healh Care Financing Review, v. 5, n. 3, p. 117-131, 1984.

LUBITZ, J.; RILEY, G. F. Trends in medicare payments in the last year of life. New England Journal of Medicine, v. 328, n. 15, p. 1092-1096, 1993.

LUNA, J. A. A emergência das doenças emergentes e as doenças infecciosas emergentes e reemergentes no Brasil. Revista Brasileira de Epidemiologia, v. 5, n. 3, 2002.

MACINKO, J.; GUANAIS, F. C.; SOUZA, M. F. M. Evaluation of the impact of the Family Health Program on infant mortality in Brazil, 1990-2002. Journal of Epidemiology \& Community Health, v. 60, n. 1, p. 13-9, 2006

MACINKO, J.; OLIVEIRA, V. B.; TURCI, M. A.; GUANAIS, F. C.; BONOLO, P. F.; LIMA-COSTA, M. F. The influence of primary care and hospital supply on ambulatory care-sensitive hospitalizations among adults in Brazil, 1999-2007. American Journal of Public Health, v. 101, n. 10, p. 1963-70, 2011. DOI: 10.2105/AJPH.2010.198887.

MARINHO, A.; MORENE, A. B.; CAVALINI, A. T. Avaliação descritiva da rede hospitalar do Sistema Único de Saúde (SUS). Brasília: Ipea, 2001 (Texto para discussão, n. 848).

MATHIAS, T. A. F.; SOBOLL, M. L. Confiabilidade de diagnósticos nos formulários de autorização de internação hospitalar. Revista de Saúde Pública, v. 32, n. 6, p. 526-532, dez. 1998.

MENDES, E. V. Uma agenda para a saúde. 2. ed. São Paulo: Hucitec, 2009.

MILLER, T. Increasing longevity and medicare expenditures. Demography, v. 38, n. 2, p. 215-226, 2001.

NEWHOUSE, J. P. Medical-care expenditure: a cross-national survey. The Journal of Human Resources, v. 12, n. 1, p.115-125, 1977.

NUNES, A. O envelhecimento populacional e as despesas do Sistema Único de Saúde. In: CAMARANO, A. A. (Org.). Os novos idosos brasileiros. Muito além dos 60 ? Rio de Janeiro: Ipea, 2004. p. 427-450. 
OMRAN, A. R. The epidemiologic transition: a theory of the epidemiology of population change. Milbank Memorial Fund Quaterly, v. 49, n. 4, p. 509-538, 1971.

PAIM, J. S. et al. O sistema de saúde brasileiro: história, avanços e desafios. The Lancet, Saúde no Brasil. 9 de maio de 2011.

PAIM, J. S. Reforma sanitária brasileira: contribuição para a compreensão e crítica. Salvador: EDUFBA; Rio de Janeiro: Editora Fiocruz, 2008.

PINHEIRO, R. S. et al. Gênero, morbidade, acesso e utilização de serviços de saúde no Brasil. Ciência e Saúde Coletiva, v. 7, n. 4, p. 687-707, 2002.

POL, L. G.; THOMAS, R. K. The demography of health and health care. 2. ed. New York: Kluwer Academic; Plenum, 2000.

RASELLA, D.; HARHAY, M. O.; PAMPONET, M. L.; AQUINO, R.; BARRETO, M. L. Impact of primary health care on mortality from heart and cerebrovascular diseases in Brazil: a nationwide analysis of longitudinal data. BMJ, v. 349:4014, 2014. DOI:10.1136/bmj.g4014.

RECHEL, B.; DOYLE, Y.; GRUNDY, E.; MCKEE, M. How can health systems respond to population ageing. World Health Organization, 2009.

RIBEIRO, M. M. Utilização de serviços de saúde no Brasil: uma investigação do padrão etário por sexo e cobertura por plano de saúde. 2005. 86 f. Dissertação (Mestrado em Demografia) - Centro de Desenvolvimento e Planejamento Regional, Universidade Federal de Minas Gerais (Cedeplar/UFMG), Belo Horizonte, 2005.

RICHARDSON, J.; ROBERTSON, L. Ageing and the cost of health services. West Heidelberg, Australia: Centre for Health Program Evaluation, 1999 (Working paper, n. 90).

RODRIGUES, C. G. Dinâmica demográfica e internações hospitalares: uma visão prospectiva para o Sistema Único de Saúde (SUS) em Minas Gerais, 2007 a 2050. Tese (Doutorado) - Centro de Desenvolvimento e Planejamento Regional, Faculdade de Ciências Econômicas, Universidade Federal de Minas Gerais (Cedeplar/Face/UFMG), Belo Horizonte, 2010.

RODRIGUES, C. G.; AFONSO, L. E. O efeito do status de sobrevivência sobre gastos com internações hospitalares públicas no Brasil em uma perspectiva temporal. Estudos Econômicos, v. 42, n. 3, p. 489-510, jul./set. 2012.

RODRIGUES-BASTOS, R. M.; CAMPOS, E. M. S.; RIBEIRO, L. C.; BASTOS-FILHO, M. G.; BUSTAMENTE-TEIXEIRA, M. T. Internações por condições sensíveis à atenção primária, Minas Gerais, 2000 e 2010. Revista de Saúde Pública, v. 48, n. 6, p. 958-967, 2014.

RUBIO, V. O. La economia em sanidad y medicina: instrumentos y limitaciones. Barcelona: Euge, 1990.

SÁ, D. A. Sistemas de informações em saúde. Departamento de Saúde Suplementar, Aula 4, Julho de 2006.

SANCHEZ, M.; VELLANKY, S.; HERRING, J.; LIANG, J.; JIA, H. Variations in Canadian rates of hospitalization for ambulatory care sensitive conditions. Healthcare Quarterly, v. 11, n. 4, p. 20-2, 2008

SESHAMANI, M.; GRAY, A. The impact of ageing on expenditures in the National Health Service. Age and Ageing, v. 31, n. 4, p. 287-294, Jul. 2002.

SILVESTRE, J. A. Por uma política pública de saúde para o idoso. In: WONG, L. R. 0 envelhecimento da população brasileira e o aumento da longevidade: subsídios para políticas orientadas ao bem estar do idoso. Belo Horizonte: Cedeplar/UFMG, 2001. p. 79-96. 
SMITH, S. D.; HEFFLER, S. D.; FREELAND, M. S. The impact of technological change on health care cost spending: an evaluation of the literature. Health Care Financing Administration. July 19, 2000.

THOE, B.; NAM, S. H. Aging risk and health care expenditure in Korea. International Journal Environmental Research and Public Health, n. 7, p. 3235-3254, 2010. Doi:10.3390/ijerph7083235.

UNITED STATES CENSUS BUREAU. Department of Commerce, 2010.

VERAS, C. M. T.; MARTINS, M. S. A confiabilidade dos dados nos formulários de Autorização de Internação Hospitalar (AIH), Rio de Janeiro, Brasil. Cadernos de Saúde Pública, v. 10, n. 3, p. 339-355, jul./set. 1994.

WIENER, J. M.; TILLY, J. Population ageing in the United States of America: implications for public programmes. Intenational Jounal of Epidemiology, v. 31, n. 4, p. 776-781, 2002.

WONG L. L. R.; CARVALHO, J. A. M. O rápido processo de envelhecimento populacional do Brasil: sérios desafios para as políticas públicas. Revista Brasileira de Estudos de População, v. 23, n. 1, p. 5-26, jan./jun. 2006.

XU, K.; SAKSENA, P.; HOLLY, A. The determinants of health expenditure: a country-level panel data analysis. World Health Organization, 2011 (Working paper).

\section{Sobre os autores}

Cristiano Sathler dos Reis é formado em Economia pela Pontifícia Universidade Católica de Minas Gerais (PUC/MG). Mestre em Demografia pelo Centro de Desenvolvimento e Planejamento Regional da Universidade Federal de Minas Gerais (Cedeplar/UFMG). Doutorando em Demografia pelo Centro de Desenvolvimento e Planejamento Regional da Universidade Federal de Minas Gerais (Cedeplar/UFMG).

Kenya V. M. S. Noronha é doutora em Economia pela Universidade Federal de Minas Gerais. Professora associada de Economia e professora do Centro de Desenvolvimento e Planejamento Regional, da Universidade Federal de Minas Gerais (Cedeplar/UFMG).

Simone Wajnman é doutora em Demografia pelo Centro de Desenvolvimento e Planejamento Regional da Universidade Federal de Minas Gerais (Cedeplar/UFMG). Professora no Cedeplar/ UFMG.

\section{Endereço para correspondência}

Cristiano Sathler dos Reis

Rua Carlos Frederico Campos, 205 - Bairro Ouro Preto

31310-400 - Belo Horizonte-MG, Brasil

Kenya V. M. S. Noronha

Faculdade de Ciências Econômicas

Departamento de Economia, Universidade Federal de Minas Gerais

Av. Antônio Carlos, 6.627 - campus Pampulha

31270-901 - Belo Horizonte-MG, Brasil

Simone Wajnman

Faculdade de Ciências Econômicas

Departamento de Demografia, Universidade Federal de Minas Gerais

Av. Antônio Carlos, 6.627 - campus Pampulha

31270-901 - Belo Horizonte-MG, Brasil 


\begin{abstract}
Population aging and hospitalization expenses of SUS: an analysis performed for Brazil between 2000 and 2010
\end{abstract}

The world population is experiencing a gradual process of aging of its age structure, due to the sharp decline in fertility and mortality in recent decades. This process, in course practically in all countries of the world, brings up the concern about health spending growth. The aim of this paper is to evaluate the effect of the changes in the age structure on hospitalization spending of the Sistema Único de Saúde (SUS) in Brazil. To achieve this goal a breakdown of health expenditures was performed using Tchoe and Nam method, adapted to the reality of the Brazilian case. The decomposition is also performed considering three groups of diseases: infectious and parasitic diseases, cancer and circulatory. The data are from the Sistema de Informação Hospitalar (SIH-SUS). In general, the results of the decomposition show the importance of variation in age structure (aging population) on SUS hospitalizations spending between 2000 and 2010, especially for groups of diseases associated with an aging population, such as circulatory diseases and cancer.

Keywords: Aging population. Spending on hospitalization. Breakdown.

\title{
Resumen
}

Envejecimiento de la población y el gasto con la hospitalización del SUS: un análisis para Brasil entre 2000 y 2010

La población mundial está experimentando un proceso gradual de envejecimiento en su estructura de edad, debido a la fuerte caída de la fecundidad y de la mortalidad en las últimas décadas. Este proceso, que ocurre prácticamente en todos los países del mundo, conlleva una gran preocupación por el crecimiento del gasto en salud. El objetivo de este artículo es evaluar el efecto de los cambios en la estructura de edades en el gasto en hospitalización del Sistema Único de Saúde de Brasil. Para lograr este objetivo, el desglose de los gastos en salud se realizó mediante el método Tchoe y Nam, adaptado para el caso de Brasil. La descomposición se hizo teniendo en cuenta tres grupos de enfermedades: infecciosas y parasitarias, cánceres y circulatorias. Los datos proceden del Sistema de Informação Hospitalar (SIH-SUS). En general, los resultados de la descomposición muestran la importancia de la variación en la estructura de edad (envejecimiento de la población) en el gasto de las hospitalizaciones en el SUS entre 2000 y 2010, especialmente para los grupos de enfermedades asociadas con el envejecimiento de la población como es el caso de las enfermedades circulatorias y el cáncer.

Palabras clave: Envejecimiento de la población. Gasto en hospitalización. Desagregación.

Recebido para publicação em 22/05/2016 Recomendado para publicação em 16/11/2016 Aceito para publicação em 04/12/2016 